\author{
Military Technical College \\ Kobry El-Kobbah, \\ Cairo, Egypt.
}

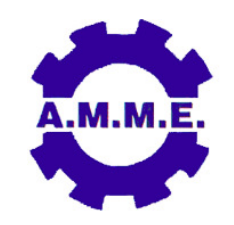

\author{
$17^{\text {th }}$ International Conference \\ on Applied Mechanics and \\ Mechanical Engineering.
}

\title{
WHEELED MOBILE ROBOT CONTROL USING ADVANCED FUZZY CONTROL
}

\author{
M. Elsayed ${ }^{\star}$, M. Sokar ${ }^{\dagger}$, S. Abd Rabbo $\ddagger$ and M. El-Arabi§
}

\begin{abstract}
Nowadays, mobile robots has a significant status in real life and industrial applications. A mathematical model of the Tricycle Mobile Robot (TMR) is introduced. A prototype of TMR with steering wheel was established. The current research presents different control techniques of TMR with auditory systems to further enhance human-robot interaction. Controlling the velocity and azimuth angle of the TMR was discussed and examined by three methods. They are Fuzzy Logic Controller (FLC) alone, fuzzy logic based on PID controller and Fuzzy Inference System (FIS) with the lookup table. All of each controller is examined with trapezoidal, triangular and Gaussian membership function, also compared with two inputs as unit step and unit sinusoidal input. The results show that the FIS with lookup table has the best output response and control signal at the sinusoidal input. Also, the minimum error signal occurs for FIS with lookup table with trapezoidal membership function at the unit step input.
\end{abstract}

\section{KEYWORDS}

Mobile robot, Modeling, Voice Recognition, Fuzzy controller, Fuzzy PID, FIS with lookup table

Assistant lecturer, Benha University, Cairo, Egypt, Mustafa.elsayed@feng.bu.edu.eg.

$\dagger \quad$ Assistant professor, Benha University, Cairo, Egypt, mohamed.sokar@feng.bu.edu.eg.

‡ Professor, Benha University, Cairo, Egypt, saberabdrabbo@feng.bu.edu.eg.

$\S$ Professor, Cairo University, Giza, Egypt, m_elarabi@eng.cu.edu.eg. 


\section{INTRODUCTION}

The main area of research on mobile robots has been activated over the last three decades. The mobile robots driving methods are differential drive, tricycle or car-like drive, synchro-drive and omnidirectional steering. The Improvements of mechanical designs and mobility control systems have helped using wheeled mobile robots in the autonomous mobile the robot applications. Wheeled mobile robots have applications in nuclear and explosives handling, warehousing, security and mobility for disabled. Although legged and treaded locomotion had been studied, and the majority of the mobile robots that studied are those, which use wheels for motion. In order to have the mobile robot perform well, two important parameters, the accuracy and reliability of its motion control should be improved. Based upon fuzzy logic, speed and steering angle control of a mobile robot are determined by goal orientation and obstacle proximity. Without keeping the track of the reliability and accuracy of robot's motion, we cannot make a robot follow a certain trajectory. If we don't have a stable and efficient controller, the mobile robot's motion cannot be controlled. Other type's mechanisms of mobile robot had been studied in Refs. [1-4].

Discussion on simulation and analysis of controller design for speed control of a DC motor actuator used in agricultural platform distinguished by [5]. It was showed that the fuzzy logic controller has better performance over conventional PID and filter compensator design for sinusoidal changes of motor speed. The effect of fuzzy membership function especially its slop for Pl-like fuzzy control system had been focused by [6]. It has a fuzzy system that consist of three membership functions. Also, it was proven that the fuzzy system confirm that the output met to the desired value. The improvements of performance of the brushless DC motor drive could be validated by changes of slops of a triangular membership function via fuzzification the error and the sum of error.

Many solutions are presented to enhance the performance of wheeled mobile robots that had a small coefficient of friction in motion. These wheeled mobile robots can endure loss of adhesion force and therefore, slide along the track conducted by [7]. On the unicycle robot, it has a fuzzy logic control on a wheeled mobile robot through wireless connection for control reported by [8], also the wireless communication is preferably on colored line tracking that intends to introduce mobile robots for handling and manipulation in warehouse environments so complicates the navigation of a swarm of mobile robots.

Other studies investigated the design of a fuzzy PID controller by using modified triangular membership functions discussed by [9]. Two useful knowledge-based fuzzy reasoning's were presented for the tracking error, and conventional triangular membership functions in fuzzy inference systems are then modified for performance improvement. Therefore, the parameters used in the proposed controllers are evaluated by using a Genetic Algorithm (GA) with a defined fitness function that is associated with the performances indices. The design of Fuzzy PID and Fuzzy PI+D controllers for different nonlinear systems was done by using 'Gaussian' Membership functions by [10]. It was observed that the response is faster with Fuzzy PID controller. However, the Fuzzy PID is exhibiting slow settling and unwanted over shoots many a times. In case of Fuzzy PI+D controller the rate of rise is slow but it is reaching final set point in less time and not producing any unwanted over shoots compared to that 
of Fuzzy PID controller. The real-world experiments involving contact in Partially Unknown Environments PUEs demonstrated that we cannot say that the fuzzy-PI controller is better than the PI controller by [11-12].

In the simulation and experimental studies significant by [13], the $\mu$-Law method was combined with simplex optimal algorithm to an automatically adjust the parameters of the structures of the FLCs. The improvements significantly the performance of FLCs through $\mu$-Law tuning method was showed by Simulation and experimental results.

As aforementioned the almost studies in mobile robot modeling and control mainly studies the differential drive on four wheels robot. There was a shortage in an implementation on modeling of tricycle mobile robot that differ from a differential drive on four wheels robot especially in the advanced application of fuzzy control also in artificial intelligence platforms. The current research apply implementation on a tricycle mobile robot called 'Sofie' and the mathematical model driven in Cartesian coordinates. Thus, in this study three controllers was built and designed to investigate the TMR prototype.

\section{METHODOLOGY}

The layout of the complete system is illustrated in Fig.1. The system mainly divided into three parts. The first part deals with signal pretreatment for recording voice signal. So, a high-quality microphone able to record sound and able to reduce noise is used. Therefore, utterance recording for training and testing must be able to filter the noise and highlight the main signal. All previous sequences must be repeated for a new command to have a clear signal to be compared with dataset in training that will happen at comparison and recognition part. The end of this part, the program able to recognize the sound and utterance of the word. The second part is the fuzzy logic control that work as brain of all the system. The input of fuzzy logic control is the feedback error and its rate of error, also the output is the velocity of robot. After this, it will be compared with the word and transfer the command to the robot with the suitable speed. The third part deals with converting a current position into location mapping related to previous position and repeated it until end of program. The Fig. 2 describe the voice signal recognition process as occurred in the robot.

A standard structure of a Fuzzy Logic Controller (FLC) is shown in Fig. 3. It consists of four stages, first, a preprocessor of the inputs that had as crisp values generated from feedback error (e) and change of error (de). Second, the fuzzification process that transform an input data to degrees of membership functions and deals with conditions of rules. At the base rule commands, the inference engine (Mamdani-type) determined the ability of a degree of functionality rules. Third, the defuzzification process of fuzzy inference output. The outputs of the fuzzy sets converted to crisp values through centroid defuzzification method. Fourth, a post-processing converts the crisp values into standard control signals. The experimental knowledge is obtained from error and change of error. Table1 include rules that relates inputs-output in the fuzzy logic controller with a trapezoidal, triangular, and Gaussian membership function and it was implemented in the simulation. The rules make control efforts based on if-then statements about (e) and (de). Each control variable is normalized into seven linguistic levels: positive big (PB), positive medium (PM), positive small (PS), zero (Z), negative 


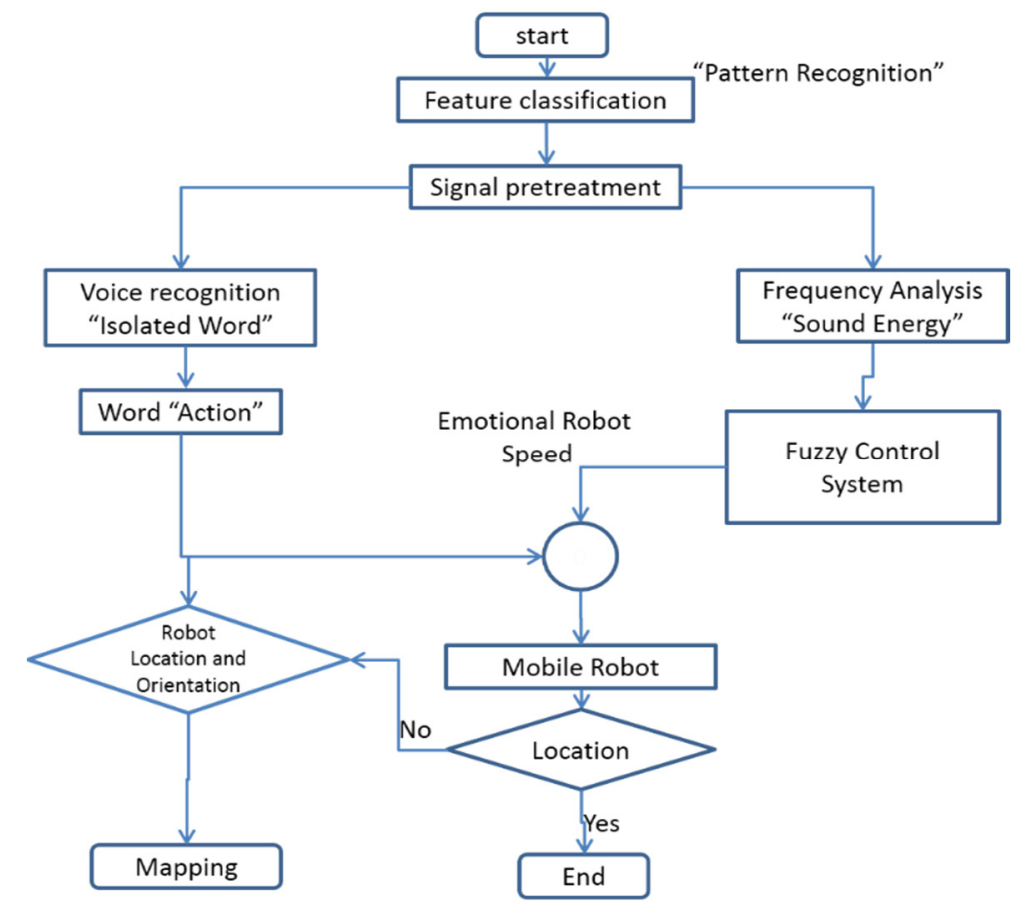

Fig. 1. Flow chart of the Algorithm.

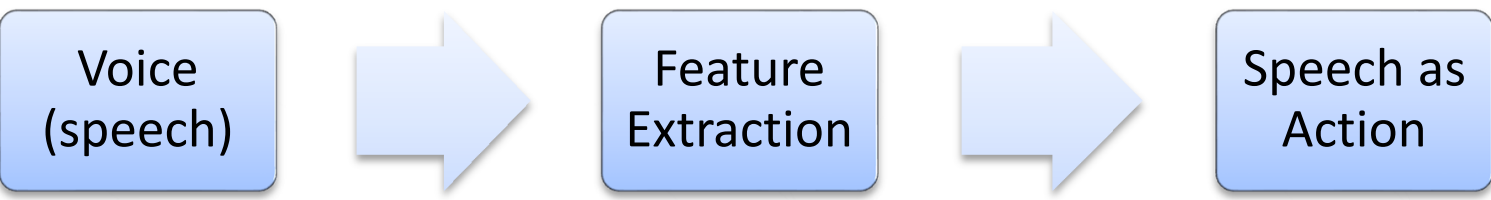

Fig.2. Block diagram of speech recognition process.

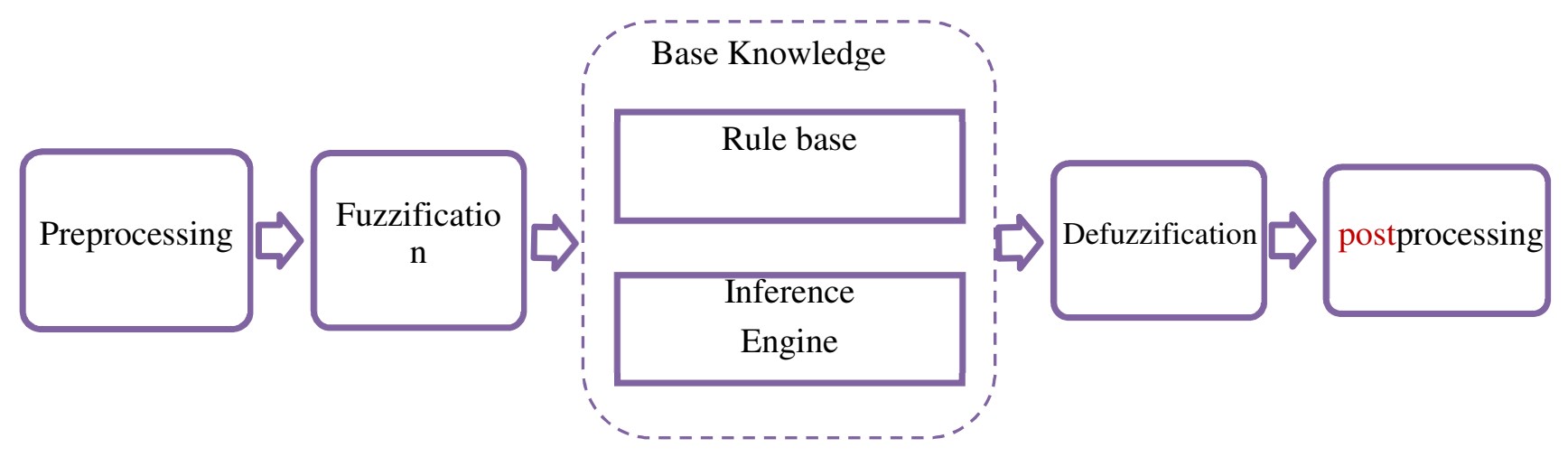

Fig.3. Block diagram of fuzzy control. 
Table 1. Trapezoidal and Gaussian rule table.

\begin{tabular}{|c|c|c|c|c|c|c|c|}
\hline$d e / e$ & NB & NM & NS & Z & PS & PM & PB \\
\hline$N B$ & PB & PB & PM & PS & PS & PS & Z \\
\hline$N M$ & PB & PM & PS & PS & Z & Z & Z \\
\hline$N S$ & PM & PM & PS & PS & Z & Z & NS \\
\hline$Z$ & PM & PS & Z & Z & Z & NS & NS \\
\hline$P S$ & PS & PS & Z & NS & NS & NM & NM \\
\hline$P M$ & PS & Z & NS & NS & NM & NB & NB \\
\hline$P B$ & Z & Z & NS & NM & NM & NB & NB \\
\hline
\end{tabular}

big (NB), negative medium (NM), and negative small (NS). The grade of each level is described by a fuzzy set. The if-then statements determined based on tests and tuning of the system. Plots of fuzzy Gaussian and trapezoidal membership functions of two inputs (e) and (de) and the output (speed) (c) are shown in Fig. 4 and Fig. 5. The mesh plot corresponding to the rules of two break-points for an FIS with lookup table controller is provided in Fig. 6 that shows the regularity of the change in the control signal.

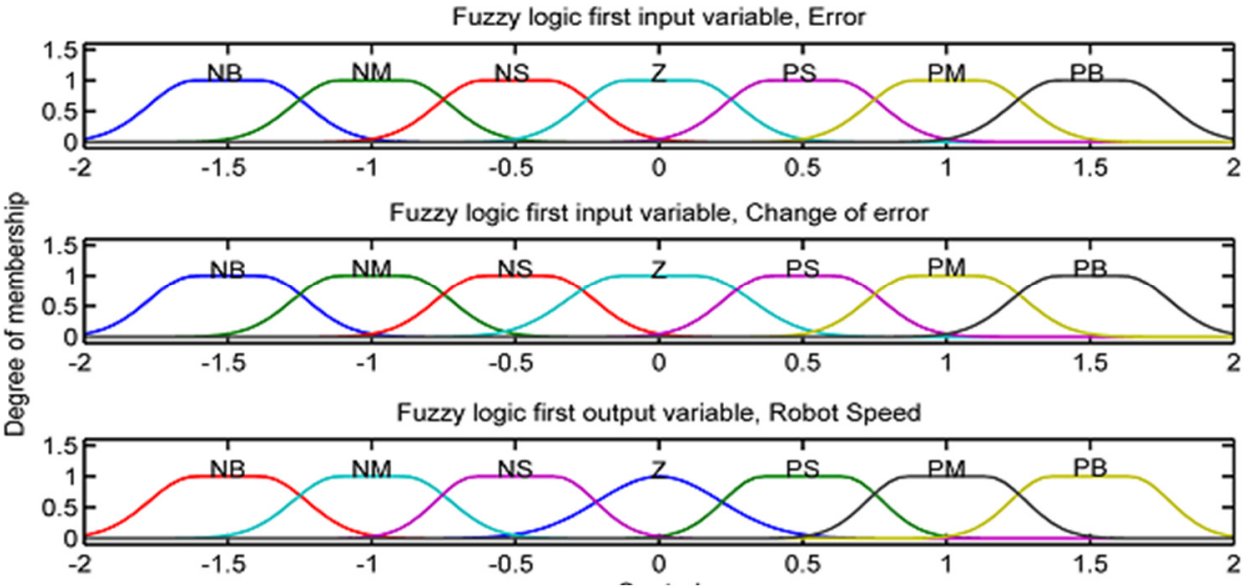

Fig. 4. Fuzzy Gaussian membership function for input and output variables.
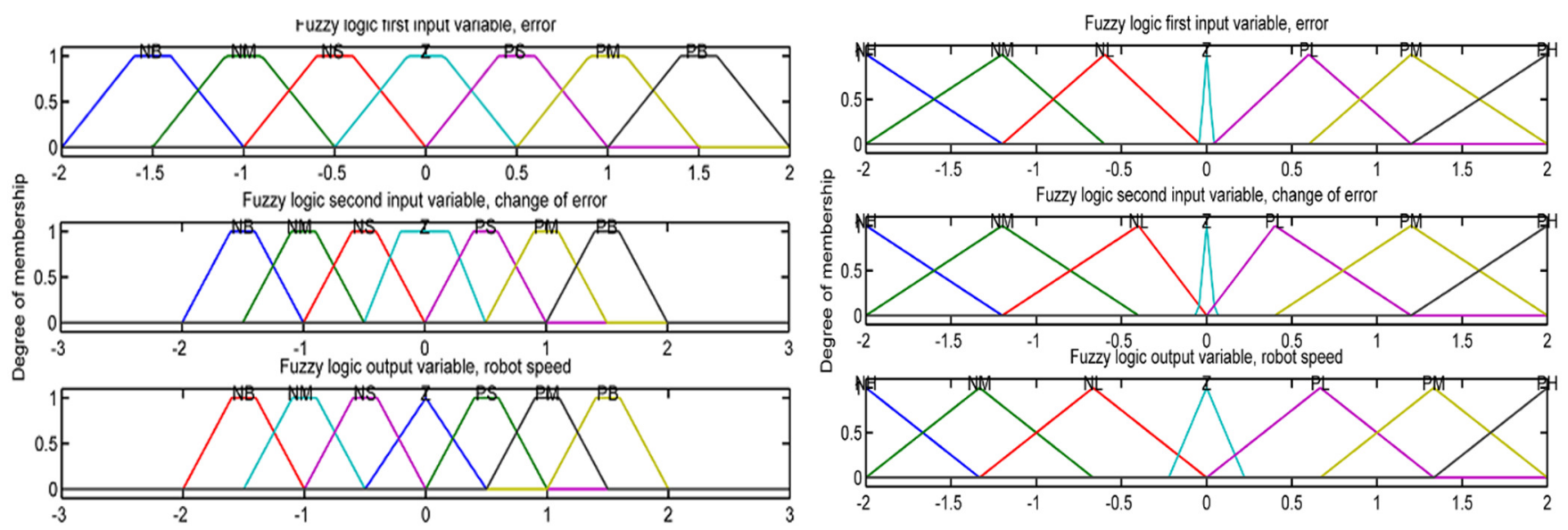

Fig. 5. Fuzzy trapezoidal and triangular membership functions for input and output variables. 


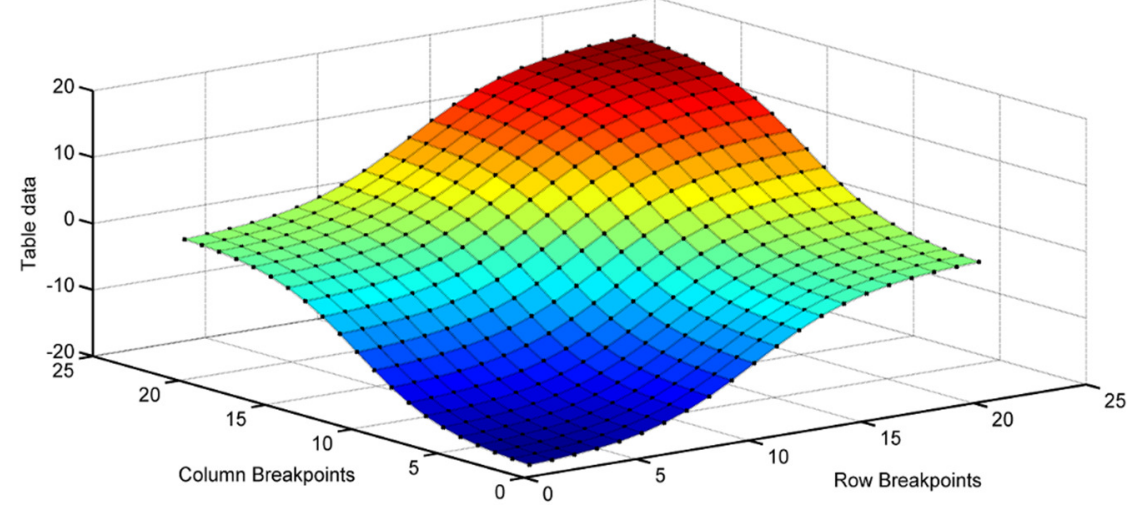

Fig. 6. Mesh plot of look up table using FIS controller.

\section{MATHEMATICAL MODEL FOR TRICYCLE MOBILE ROBOT}

The prototype of a tricycle type robot driven by two rear wheels autonomously and a one front wheel as a castor called "Sofie Robot" as shown in Fig. 7. The kinematic model of a nonholonomic constraint of pure rolling and without slipping based on the configuration shown in Fig. 8. If the position of wheeled mobile is denote by $x(t)$ and $y(t)$ along the $X$ and $Y$ Cartesian coordinate frames and it called robot initial frame

$R(t)=\frac{d}{\tan \alpha}$

$\dot{\theta}=\omega_{t}=\frac{v_{s}}{d} \sin \alpha=\frac{\omega_{s} r}{d} \sin \alpha$

Where, $\dot{x}$ and $\dot{y}$ are components of forward velocities; $\theta(t)$ represents the angle between the heading direction and the x-axis. The robot rotates with an angular speed $\omega_{t}$ around the Instantaneous Center of Rotation (ICR) whose distance $\mathrm{R}$ to the robot center axis. Also, $v_{s}, \omega_{s}$ and $r$ are respectively the linear speed of the front wheel, its angular speed and wheel radius.

Kinematic model in the robot body frame:

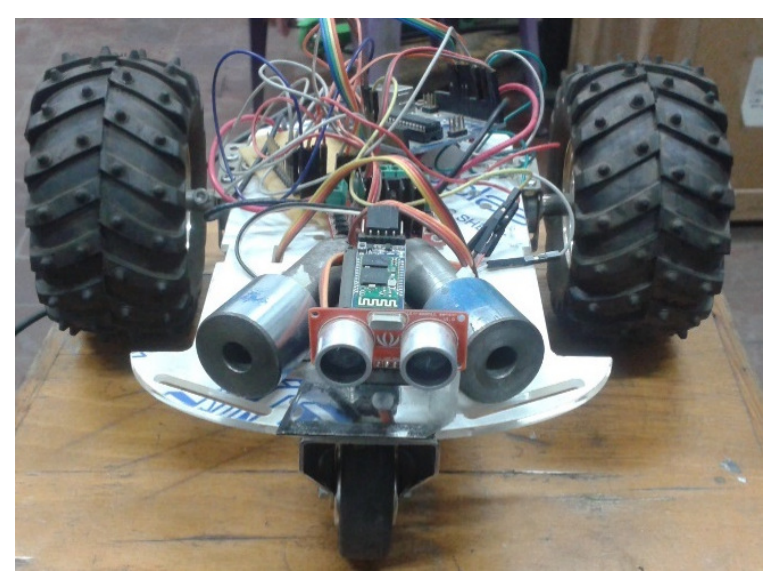

Fig. 7. Sofie robot.

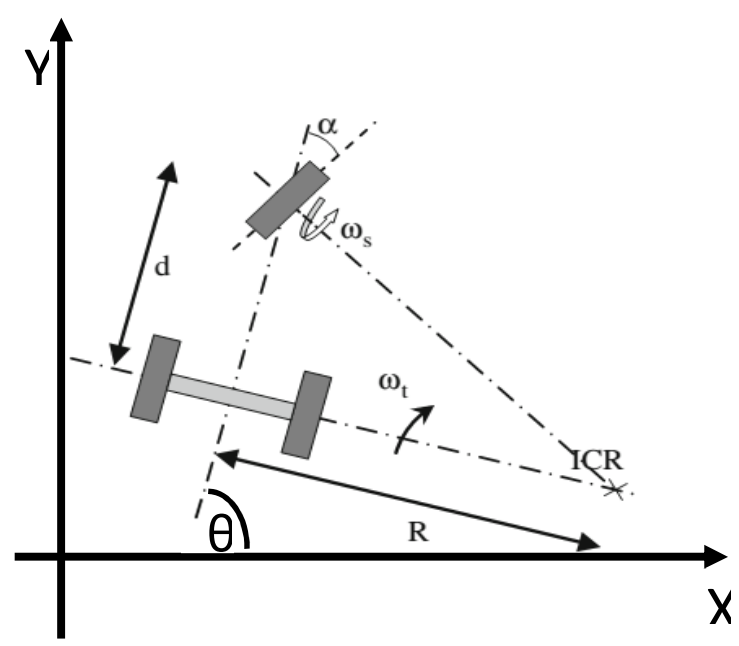

Fig. 8. Kinematics of tricycle mobile robot. 


$$
\left[\begin{array}{c}
v_{x} \\
v_{y} \\
\dot{\theta}
\end{array}\right]=\left[\begin{array}{c}
\cos \alpha \\
0 \\
\sin \alpha \\
\hline d
\end{array}\right]\left[v_{s}\right]
$$

Kinematic model in the initial frame:

$$
\begin{gathered}
v_{t}=v_{s} \cos \alpha \\
\dot{x}=v_{s} \cos \alpha \cos \theta \\
\dot{y}=v_{s} \cos \alpha \sin \theta \\
{\left[\begin{array}{c}
\dot{x} \\
\dot{y} \\
\dot{\theta}
\end{array}\right]=\left[\begin{array}{cc}
\cos \theta & 0 \\
\sin \theta & 0 \\
0 & 1
\end{array}\right]\left[\begin{array}{c}
v_{t} \\
\omega_{t}
\end{array}\right]}
\end{gathered}
$$

The simulation results based on state space model (open loop transfer function) of the TMR at unit step input. Although the open loop system in Fig. 9 shows stability in nature, but the closed loop step response indicates a demand for a controller to improve rise time, settling time, overshoot. The TMR response for each three parameters of the position $(X, Y, \theta)$ is shown in Fig.10, also distinguished from given transfer function of TMR. Also, characteristic parameters of TMR at world coordination are given in the Table 2.

Open loop transfer function without controller

$$
\frac{5.518 s^{2}+7.747 s+2.668}{s^{3}+0.4481 s^{2}}
$$

Closed loop transfer function without controller

$\frac{5.518 s^{2}+7.747 s+2.668}{s^{3}+5.966 s^{2}+7.747 s+2.668}$
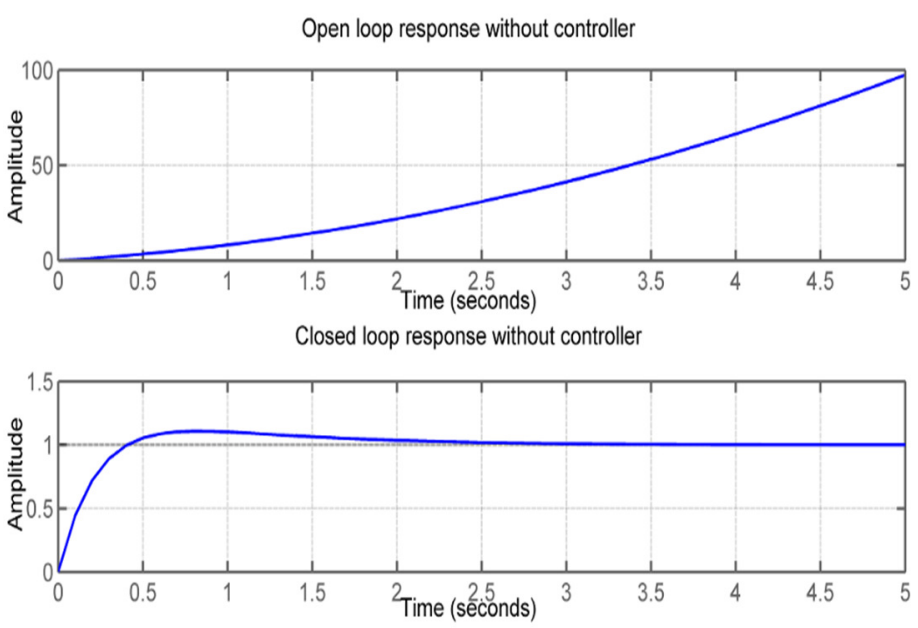

Fig. 9. TMR response without controller.

The given model examined by three controllers where simulated in MATLAB/Simulink@ with block diagram shown in Fig.11. A signal generator produces an input reference of step and sinusoidal functions for each control blocks. The transfer function of controller implemented in the simulation followed by the Tricycle mobile robot model. In the current research the control system for Sofie robot consists of three parts (input signals - Controller - Plant "Robot") as shown in Fig.12. The controller of system is based on 
Table 2. Characteristic parameters of TMR.

\begin{tabular}{|l|c|}
\hline \multicolumn{1}{|c|}{ Parameter } & Value \\
\hline damping ratio, $\zeta$ & 0.5169 \\
\hline natural frequency, $\omega_{\mathrm{n}}[\mathrm{rad} / \mathrm{s}]$ & 38.6899 \\
\hline head angle (steering angle), $\alpha$ & $45^{\circ}$ \\
\hline initial angle (absolute angle), $\theta$ & $90^{\circ}$ \\
\hline
\end{tabular}

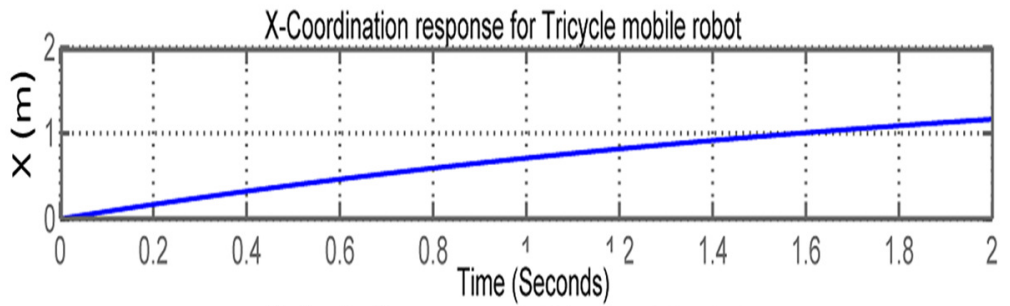

Y- Coodination response for Tricycle mobile robot
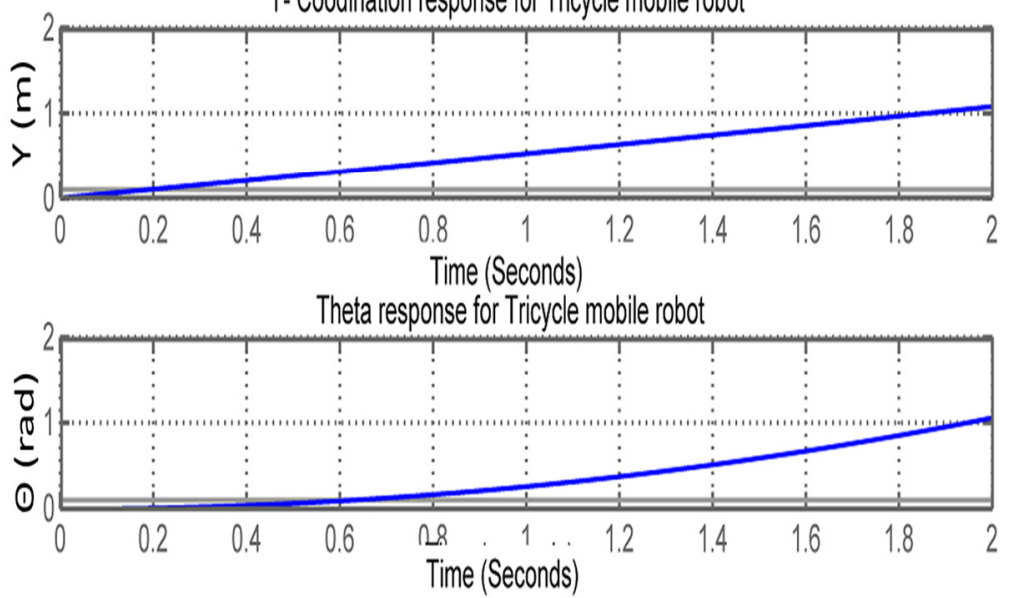

Fig. 10. Plots of response for Tricycle mobile robot.

Fuzzy logic control that each controller is examined by three types of membership function explained earlier for the two types of input signals respectively. The system is examined against type of controllers, the first one is Fuzzy logic controller in a closed loop control system. The second one is the Fuzzy-PID system with input derivative gain and output integral gain. The third type is the Fuzzy inference system (FIS) with lookup table with two break points.

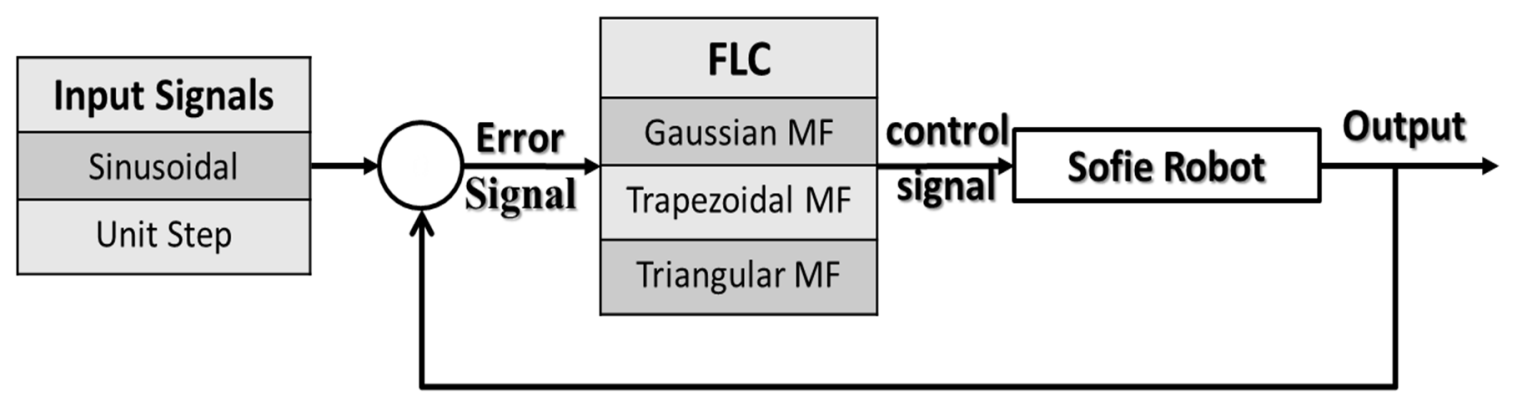

Fig. 11. A typical control system. 


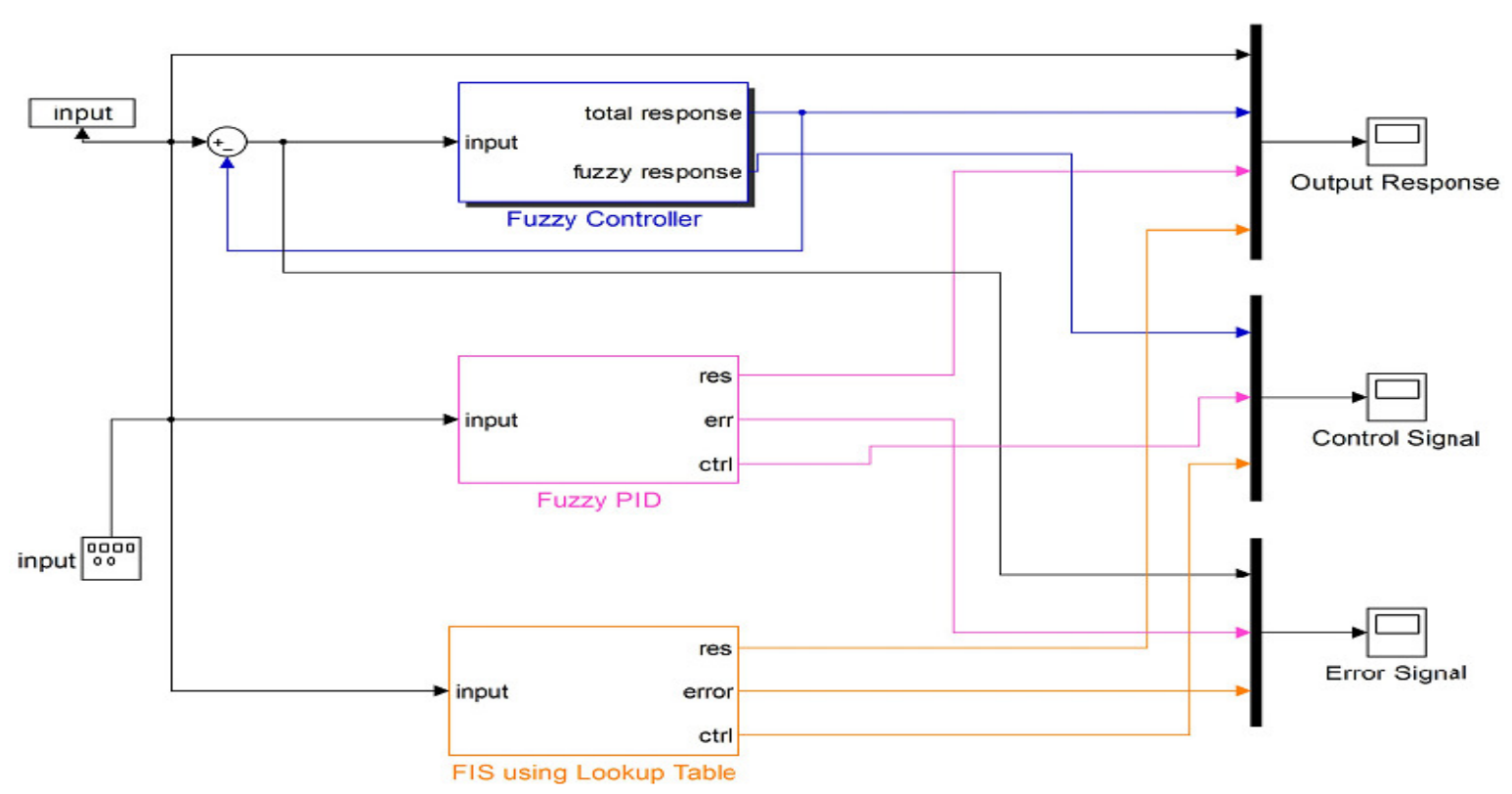

Fig. 12. Simulink model of different type's controller.

The corresponding defuzzification sub-block is shown in Fig.13. The aim of a controller is to maintain a plant in a specific situation, by control a set of variables and selecting the sufficient control actions. The Fuzzy-PID controller works like its classical homonym, but the input variables and the control action are the linguistic terms in fuzzy controller. Explanation of the influence of tuning parameter allowed by an analytical development of fuzzy controllers on the system. The new model design for implementing the fuzzy inference system by adding a PID controller on Fuzzy model illustrated in Fig.14. The value of gains in terms of a PID is trained by trial and error method. A last one controller consists of lookup table fuzzy inference system with two break points of two input variables as shown in Fig.15.

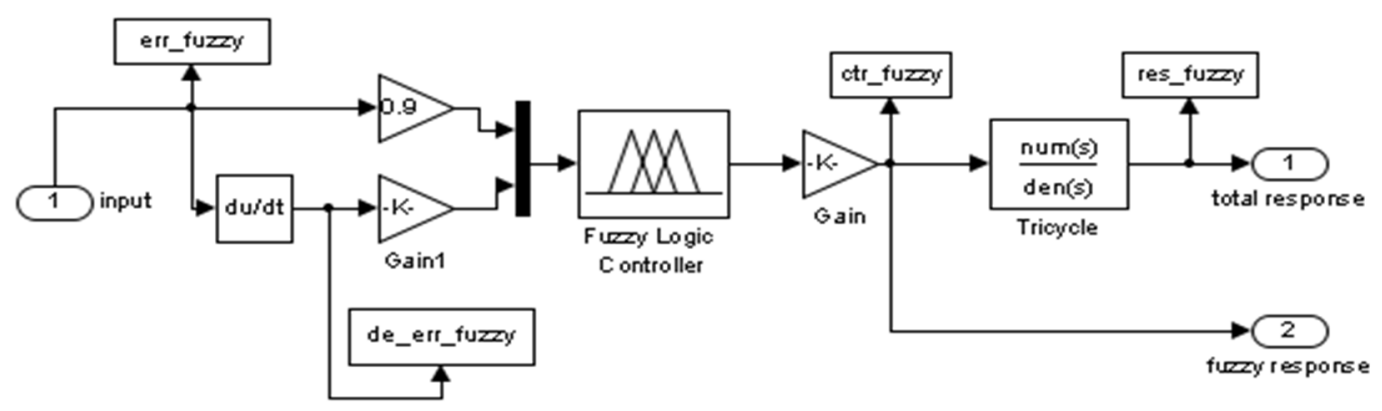

Fig. 13. Simulink model of fuzzy controller.

\section{RESULTS AND DISCUSSION}

Based on simulation, the input was (unit step, unit ramp and sinusoidal input) examined with trapezoidal, triangular and Gaussian membership function. Fig.16 and Fig. 17 


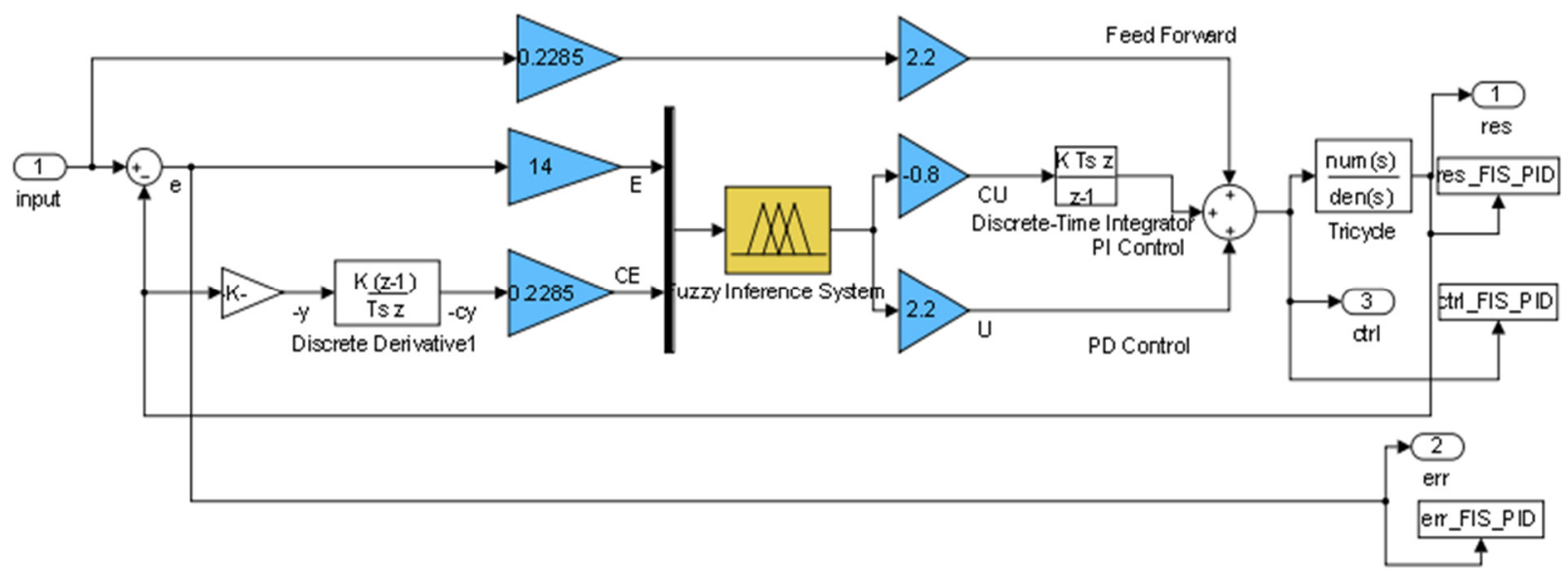

Fig. 14. Simulink model of fuzzy-PID controller.

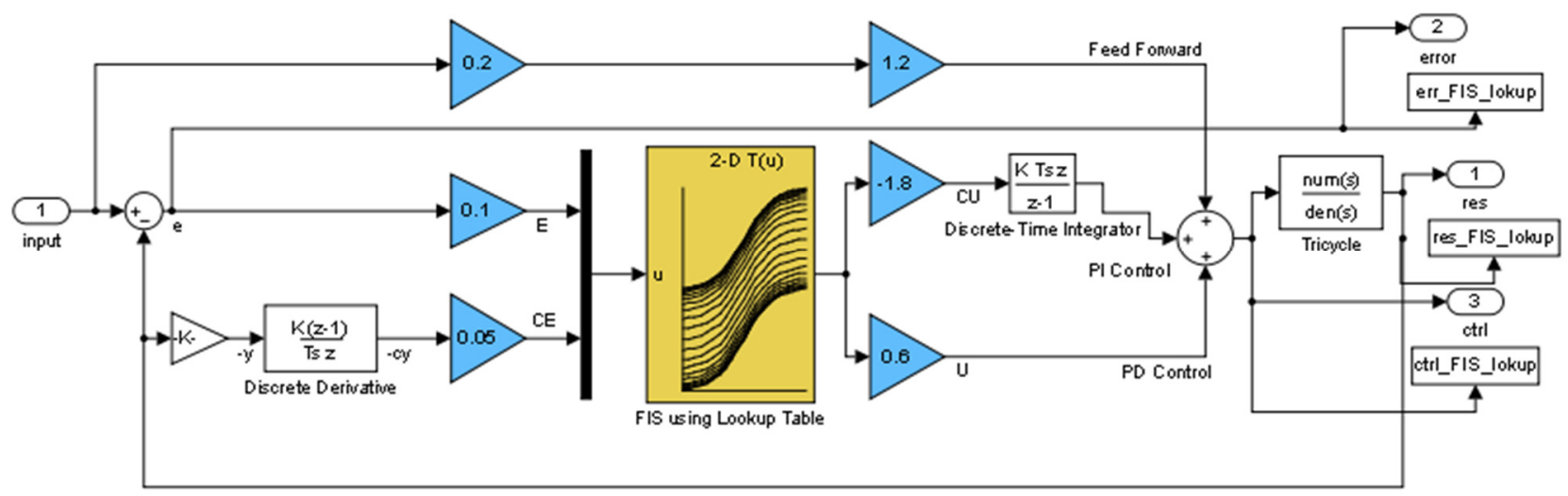

Fig. 15. Simulink model of fuzzy using look up table controller.
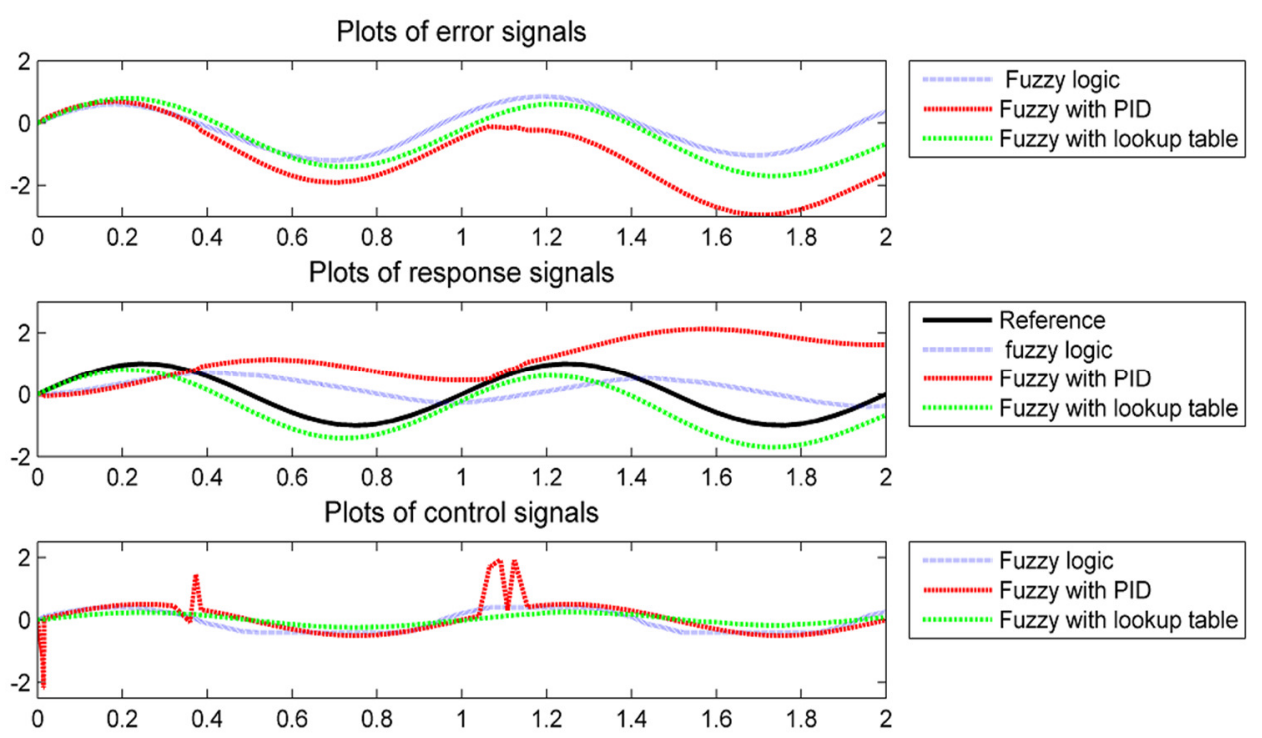

Fig. 16. Plots Fuzzy, Fuzzy-PID and FIS with lookup-table error, response and control signal with trapezoidal membership function at Sinusoidal input. 

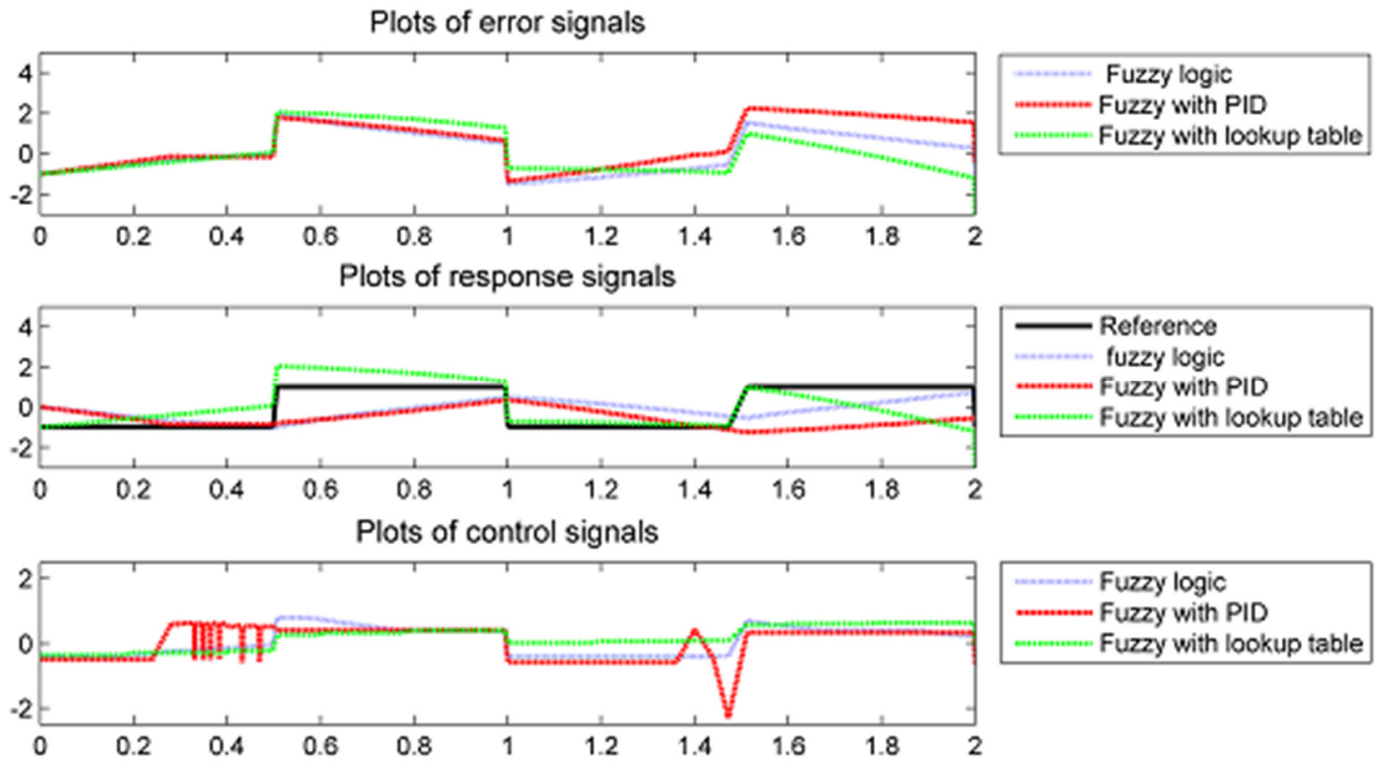

Fig. 17. Plots Fuzzy, Fuzzy-PID and FIS with lookup-table error, response and control signal with trapezoidal membership function at Step input.

plots fuzzy, fuzzy-PID and FIS with lookup-table for trapezoidal membership function at sinusoidal and unit step inputs respectively. For sinusoidal input, the FIS with lookup table has the best output response. The FLC and FIS with lookup table have the similar error range. For unit step input the FLC and fuzzy-PID have the output response like the first order response but, in FIS with lookup table has scattered in its output response. The FLC and fuzzy-PID have the similar error range and change error rate.

Figures18 and 19 plots fuzzy, fuzzy-PID and FIS with lookup-table for triangular membership function at sinusoidal and unit step inputs respectively. For sinusoidal input the fuzzy-PID and FIS with lookup-table have the best output response. Therefore, the FIS with lookup-table has the best performance. The fuzzy-PID has the Minimum error range. For unit Step input the FIS with lookup-table has the nearest output response. The fuzzy-PID and FIS with lookup-table have the similar error range.

Figures 20 and 21 plots fuzzy, fuzzy-PID and FIS with lookup-table for Gaussian membership function and sinusoidal at unit step inputs respectively. For sinusoidal input the FIS with lookup table has the best output response. Also, FLC and FIS with lookup table have the similar error range. For unit step input the FLC and fuzzy-PID have the output response like the first order response but, in FIS with lookup table has scattered in its output response. The fuzzy-PID with Gaussian MF has been minimum error signal and smoother amplitude from suddenly changes also change a direction of signals in trapezoidal from Negative side direction to the positive side by Gaussian MF. Also, the results proved that the FIS with lookup table has the best output response and a control signal at the sinusoidal input. 


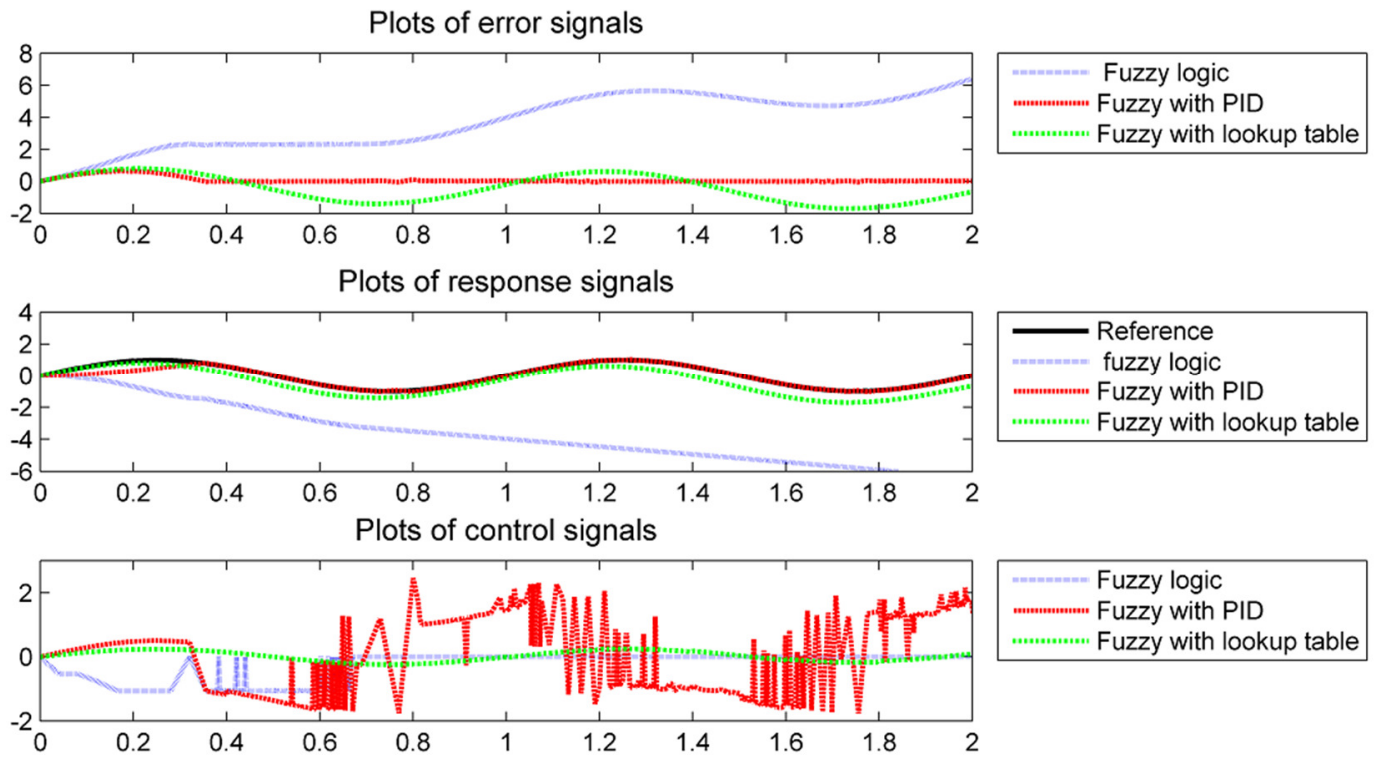

Fig. 18. Plots Fuzzy, Fuzzy-PID and FIS with lookup-table error, response and control signal for triangular membership function at Sinusoidal input.

Plots of error signals
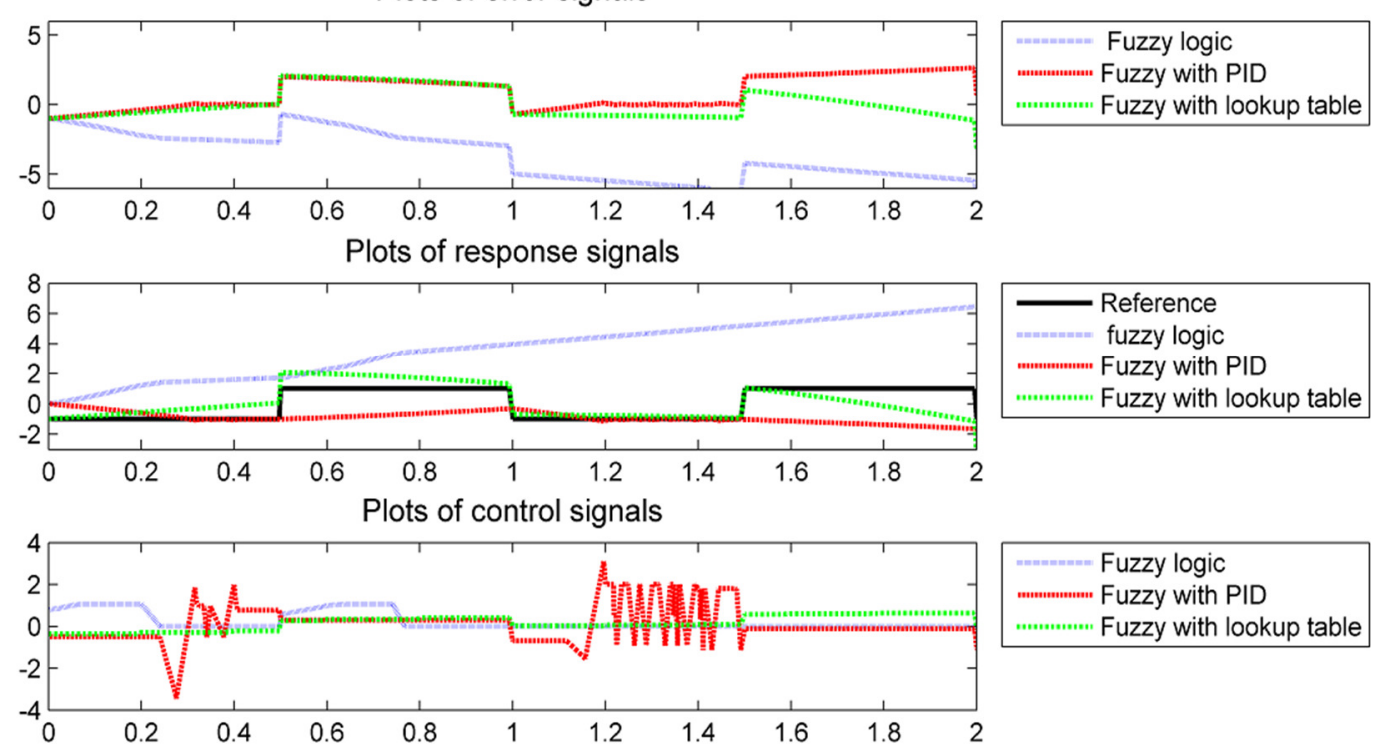

Fig. 19. Plots Fuzzy, Fuzzy-PID and FIS with lookup-table error, response and control signal with triangular membership function at step input 

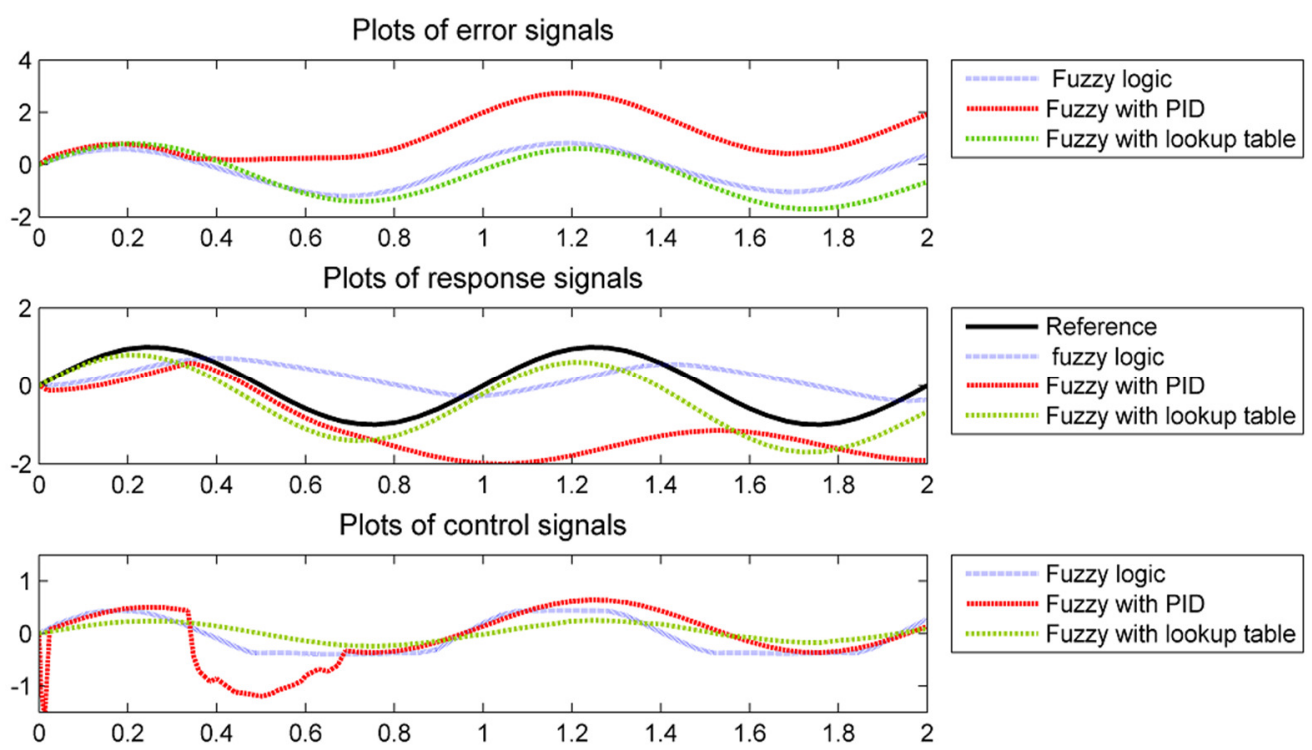

Fig. 20. Plots Fuzzy, Fuzzy-PID and FIS with lookup-table error, response and control signal with Gaussian membership function at Sinusoidal input.
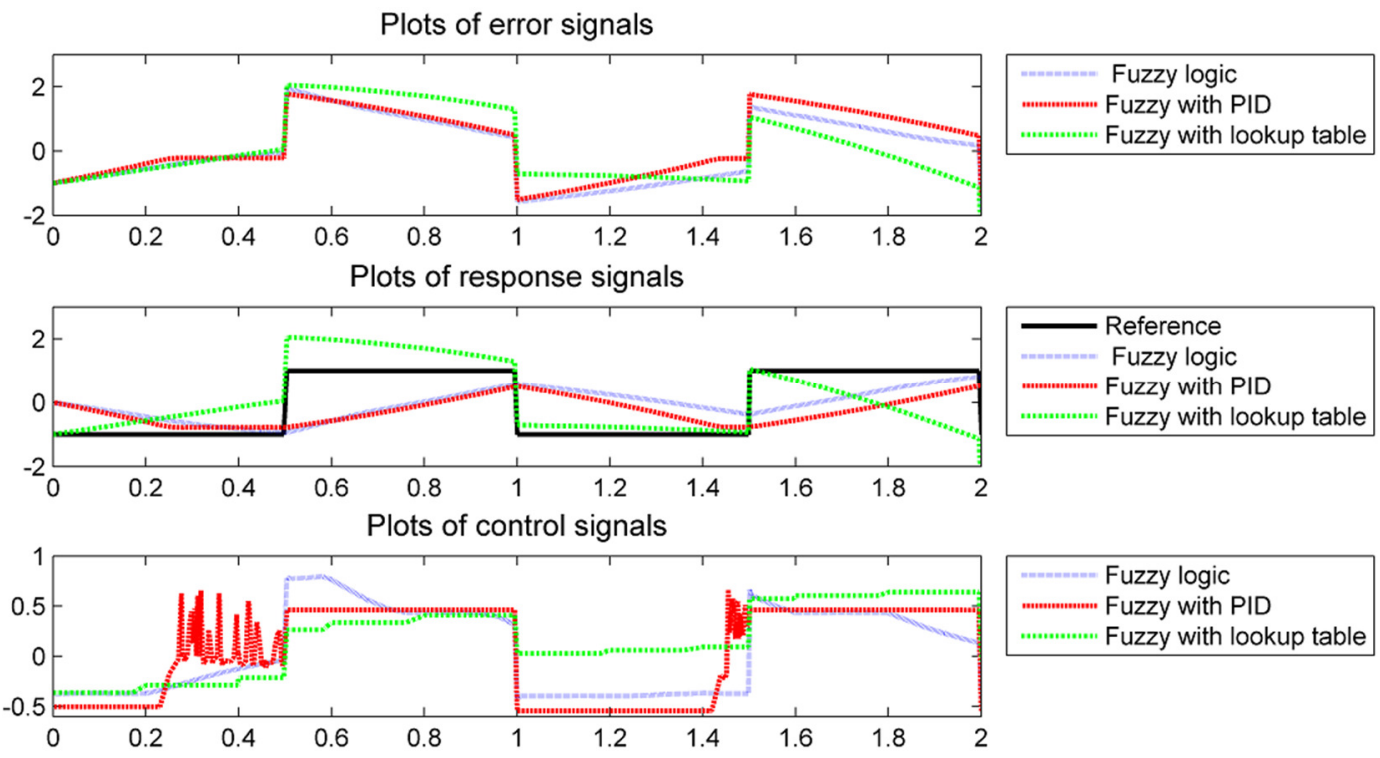

Fig. 21. Plots Fuzzy, Fuzzy-PID and FIS with lookup-table error, response and control signal with Gaussian membership function at step input. 


\section{CONCLUSION}

The Tricycle Mobile Robot with steering wheel was built as a prototype. It has a variable traveling speed and steering mechanism by servo motor and without differential motion control. Also, it defined as autonomous robot with emergency stopping. The given model is divided into two part. First, the mathematical model of tricycle mobile robot was built as an open loop transfer function. Second, the control part which based on Fuzzy logic control system such, Fuzzy controller, Fuzzy controller along PID gains and an FIS with lookup table. All of each controller are examined with Trapezoidal, Triangular and Gaussian membership function. This work proved that at the Sinusoidal input an FIS with lookup table has the best output response, where it is always has a minimum deviation about reference input signal through a long period of time compared with other tested controller features and control signal. Although, the Minimum error signal occur FIS with lookup table with Trapezoidal membership function at unit step input. It has more benefits than the other controller FLC and FuzzyPID. The given Fuzzy-PID will be provided with more enhancement with Genetic Algorithms to eliminate the response error.

\section{REFERENCES}

[1] Muniandy, M., \& Muthusamy, K. (2012). An Innovative Design to Improve Systematic Odometry Error in Non-holonomic Wheeled Mobile Robots. Procedia Engineering, 41(Iris), 436-442. http://doi.org/10.1016/j.proeng.2012.07.195.

[2] Rodríguez, N. E. N. (2011). Advanced Mechanics in Robotic Systems.

[3] Tzafestas, S. G. (2014). Introduction to Mobile Robot Control. http://doi.org/10.1016/B978-0-12-417049-0.00001-8.

[4] Wang, B., Li, Z., \& Ding, N. (2011). Speech control of a teleoperated mobile humanoid robot. IEEE International Conference on Automation and Logistics, ICAL. http://doi.org/10.1109/ICAL.2011.6024739.

[5] Shamshiri, R., Ishak, W., \& Ismail, W. (2013). Design and Simulation of Control Systems for a Field Survey Mobile Robot Platform, 6(13), 2307-2315.

[6] Jang, B. J. B., Im, S. I. S., \& Jung, I. J. I. (2009). Optimal tuning of PI-like fuzzy controller using variable membership function's slope. 2009 IEEE International Symposium on Assembly and Manufacturing. http://doi.org/10.1109/ISAM.2009.5376964.

[7] Fernández, R., Aracil, R., \& Armada, M. (2012). Control de Tracción en Robots Móviles con Ruedas. Revista Iberoamericana de Automática E Informática Industrial RIAI. http://doi.org/10.1016/j.riai.2012.09.008.

[8] Faisal, M., Hedjar, R., Al Sulaiman, M., \& Al-Mutib, K. (2013). Fuzzy logic navigation and obstacle avoidance by a mobile robot in an unknown dynamic environment. International Journal of Advanced Robotic Systems, 10. http://doi.org/10.5772/54427.

[9] Juang, Y.-T., Chang, Y.-T., \& Huang, C.-P. (2008). Design of fuzzy PID controllers using modified triangular membership functions. Information Sciences, 178(5), 1325-1333. http://doi.org/10.1016/j.ins.2007.10.020.

[10] Ananthababu, P., Reddy, B. a, \& Charan, K. R. (2009). Design of Fuzzy PI+ D and Fuzzy PID Controllers Using Gaussian Input Fuzzy Sets, 957-961. 
[11] Mendes, N., Neto, P., Pires, J. N., \& Loureiro, A. (2013). An optimal fuzzy-PI force/motion controller to increase industrial robot autonomy. International Journal of Advanced Manufacturing Technology, 68(1-4), 435-441. http://doi.org/10.1007/s00170-013-4741-3.

[12] Santos, M., Dormido, S., \& Cruz, J. M. De. (1996). Fuzzy-PID Controllers vs . Fuzzy-PI Controllers. Fuzzy Systems, Proceedings of the Fifth IEEE International Conference, $3(34), \quad 1598 \quad-\quad 1604$. http://doi.org/10.1109/FUZZY.1996.552571.

[13] Zhuang, H., \& Wang, Y. (2006). Knowledge-based Tuning II: $\mu$-Law Tuning of a Fuzzy Lookup Table. In Advanced Fuzzy Logic Technologies in Industrial Applications (pp. 67-81). 